\title{
Improving machine dynamics via geometry optimization
}

\author{
Lucian Tudose · Cristina Stănescu • András Sóbester
}

Received: 24 May 2010 / Revised: 24 January 2011 / Accepted: 18 February 2011

(C) Springer-Verlag 2011

\begin{abstract}
The central thesis of this paper is that the dynamic performance of machinery can be improved dramatically in certain cases through a systematic and meticulous evolutionary algorithm search through the space of all structural geometries permitted by manufacturing, cost and functional constraints. This is a cheap and elegant approach in scenarios where employing active control elements is impractical for reasons of cost and complexity. From an optimization perspective the challenge lies in the efficient, yet thorough global exploration of the multi-dimensional and multi-modal design spaces often yielded by such problems. Moreover, the designs are often defined by a mixture of continuous and discrete variables-a task that evolutionary algorithms appear to be ideally suited for. In this article we discuss the specific case of the optimization of crop spraying machinery for improved uniformity of spray deposition, subject to structural weight and manufacturing constraints. Using a mixed variable evolutionary algorithm allowed us to optimize both shape and topology. Through this process we have managed to reduce the maximum roll angle of the sprayer by an order of magnitude, whilst
\end{abstract}

L. Tudose · C. Stănescu

Faculty of Machine Building, Dept. of Machine Elements and

Tribology, Technical University of Cluj-Napoca, Bd. Muncii 103-105, 400641, Cluj-Napoca, Romania

L. Tudose

e-mail: Lucian.Tudose@omt.utcluj.ro

C. Stănescu

e-mail: Cristina.Stanescu@omt.utcluj.ro

A. Sóbester $(\varangle)$

School of Engineering Sciences, University of Southampton,

Southampton, SO17 1BJ, UK

e-mail: a.sobester@ soton.ac.uk allowing only relatively inexpensive changes to the baseline design. Further (though less dramatic) improvements were shown to be possible when we relaxed the cost constraint. We applied the same approach to the inverse problem of reducing the mass while maintaining an acceptable roll angle-a $2 \%$ improvement proved possible in this case.

Keywords Shape optimization - Topology optimization · Evolutionary algorithms $\cdot$ Suspension design

\section{Introduction}

Recent decades have seen the emergence of a broad range of computational methods for structural optimization. These include techniques that enable the exploration of design spaces that are not bounded by conventional design variable range constraints - indeed, they do not require the definition of design variables in the conventional sense. Instead, in this class of heuristics an optimal (though not necessarily globally optimal) design emerges from a continuum or, in the case of frameworks, from a densely populated structural universe (for a recent discussion of the potential and the pitfalls of these methods see Huang and Xue (2010)). The motivation for the present study is that a vast number of structural design problems still defy solution through these means for at least two possible reasons.

First, the range of possible objective functions that variable-free shape and topology search engines have been developed for is limited. Indeed, in some cases it is not even clear what the underlying goal function of these methods is, as often they merely offer a heuristic that defines a path through the design space, based on some iteration rule, such as 'at each iteration remove the most lightly loaded element'. Where an objective is in evidence, it is usually a 
static one, such as minimal compliance or minimum stress, as the evolutionary approaches designed around other objectives can become exceedingly complicated (Sigmund 2001) (though certain dynamic instances are beginning to emerge Zhu et al. 2007).

Second, heuristics based on continua are often illequipped to cope with all but the simplest constraints. While Kreisselmeier-Steinhauser-type aggregation functions (Martins and Poon 2005; París et al. 2009, 2010) have been used to deal with stress constraints, in many 'real-life' scenarios limits on complexity, constraints related to manufacturing, etc. have to be imposed and, to the best of our knowledge, constraint formulations for these are yet to be developed for topology optimization algorithms.

Here we tackle a design problem that eludes such parameter-free approaches on all three counts. As we are about to see, the objective function is relatively complex and dynamic in nature and it is not directly linked to any of those goals that drive parameter-free heuristics. Moreover, restrictions on tooling and manufacturing cost impose relatively awkward geometrical constraints. More specifically, we consider the problem of improving the dynamic performance of a crop spraying machine-minimizing the rolling motion of the long spraying boom translates into improved uniformity of spray deposition. Tooling constraints mean drastic limitations on the changes permitted to the geometry of the boom and its suspension mechanism.

Another defining feature of our problem is that the design is described by both discrete and continuous parameters. Hence, gradient-based exploitation of the basins of attraction of the design space is not feasible. We are therefore limited to zeroth order, direct searches. Additionally, as we shall see, the definition of the ranges of these variables also contains a twist, which further restricts the range of applicable optimization technologies.

Evolutionary algorithm optimization has some history in the context of exploring the space of structures of a specified layout in the search for the shape with the most favourable dynamic response. An early instance of passive vibration control through evolutionary algorithm shape optimization can be found in a paper by Keane (1995), where the lengths of the bars making up a satellite structure were optimized, yielding an unusual-looking structure, which minimized the vibration energy transmitted in a certain frequency bandspacecraft engineering is, incidentally, still one of the most active areas in terms of shape design for vibration attenuation (see Kamesh et al. 2010 for a recent study) and is therefore likely to benefit from advances in this type of optimization technology. Another active field is that of seismic engineering-for example, Oka et al. (2008) recently used an evolutionary algorithm to optimize the design of hysteretic dampers connecting adjacent structures. A genetic search engine was recently the tool of choice in the marine vessel structural dynamics study reported by Dylejkoa et al. (2007) too- - they sought to optimize a resonance changer to minimize the vibration transmission in a submarine.

There is, thus, evidence in a variety of fields that evolutionary algorithm searches can be effective in this type of structural design application. Additionally, the requirement for handling a mixed variable set, as well as for keeping a global outlook across a potentially multi-modal design space, makes evolutionary algorithms almost uniquely suited to this application. We have therefore selected this class of heuristics for the three optimization studies discussed in this paper. In terms of the actual implementation of the algorithm, we opted for Cambrian (Tudose et al. 2010), a tool incorporating numerous recent developments in evolutionary search technology, whose name hints at the inclusion of the paradigm of punctuated equilibra ${ }^{1}$ in the design of its underlying heuristic. The periods of equilibrium serve as spells of evolution driven exclusively by the selective pressure of the constraints of the problem-as we shall see, effective constraint handling is a feature one of our optimization studies will call upon. Here are, in a nutshell, the key steps of the two epoch algorithm.

- 1. EPOCH ONE: Evolution towards feasibility. Simulate one generation of artificial evolution driven exclusively by the selective pressure of the constraints.

- 2. Feasibility test. Does the ratio of feasible individuals exceed a pre-set threshold value? If not, return to 1 .

- 3. EPOCH TWO: Evolution towards high performance and feasibility. Simulate one generation of artificial evolution (following the canonical template of Goldberg 1989) driven by the objective function penalised by the constraints (this step is equivalent to the standard constrained evolutionary search, based on the fundamental principles introduced by Fiacco and McCormick 1968-our implementation follows the guidelines of Keane and Nair 2005).

- 4. If an objective-related convergence criterion is met, stop. Otherwise, if the percentage of feasible individuals has dropped below a certain threshold, return to 1 ; if not, return to 3 .

We shall now introduce the design problem at the centre of this paper in detail (Section 2), after which we proceed to discuss its structural dynamics aspects (Section 3), followed by its solution in three separate stages (Sections 4-6), each representing a different level of design parameterisation flexibility and different cost implications. We conclude the discussion with reflections on possible extensions to the study, as well as possible implications in other areas of engineering design.

\footnotetext{
${ }^{1} \mathrm{~A}$ phenomenon associated by some biologists with the so-called Cambrian explosion, a time of rapidly increasing genetic diversity.
} 
Fig. 1 The spraying machine on the standardized rough track

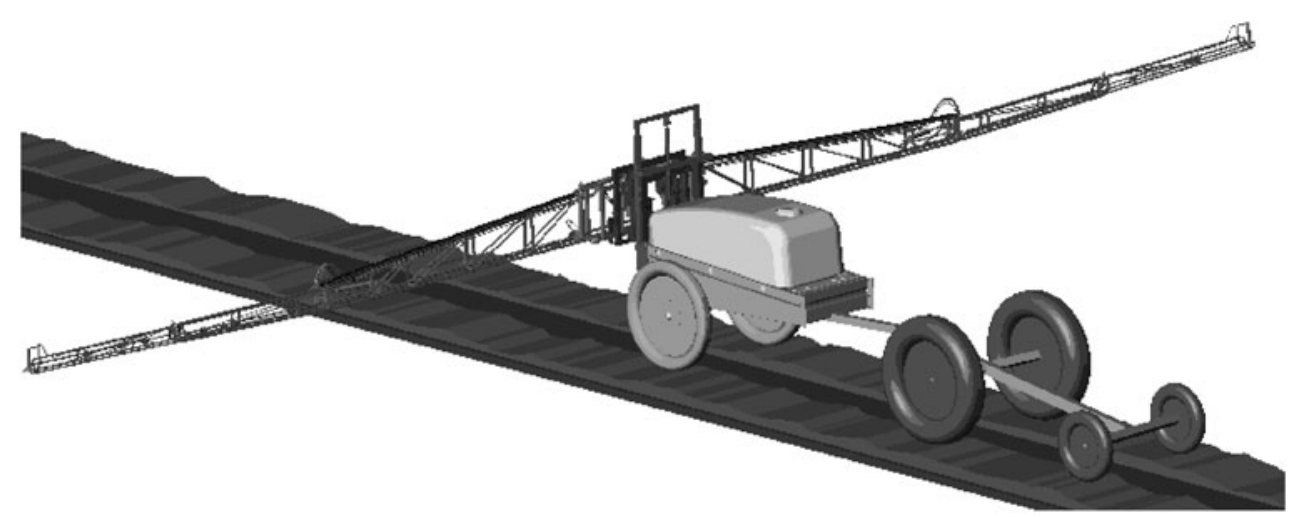

\section{The design problem}

The subject of our design study is a trailed crop spraying machine (Fig. 1), which, for the purposes of the evaluation of the objectives and constraints of the design problem, is assumed to be moving along a standardized, rough track (representing a freshly ploughed field, as per ISO 5008:2002 (ISO 2002)), at a speed of $4 \mathrm{~km} / \mathrm{h}$. The standard defines the track in the form of two strips of different elevation profiles, one to be followed by each wheel (Fig. 2). The asymmetry of the track translates into a rolling motion of the main body of the spraying machine-we are interested in the rolling motion transmitted by the main body to the spraying boom, which is mounted on a suspension designed to reduce this. We shall neglect any yawing and pitching components to the motion of the boom. Further, we shall assume the boom to be rigid. Incidentally, this latter assumption is confirmed as reasonable by the manufacturer of the machine (based on empirical observations) and by Parloo et al. (2005), Clijmans et al. (2000), Langenakens et al. (1999) and Anthonis et al. (2005) on the basis that the resonant frequency of vertical boom suspensions for long booms is generally of the order of $0.1 \mathrm{~Hz}$ and the first flexible vertical mode is usually located in the range between $0.6-1.5 \mathrm{~Hz}$.
The design 'wish-list' the following work is based on has three key entries. First, the maximum rolling angle of the boom must be minimized, or at least kept within limits defined by spray deposition quality constraints. Second, the mass of the boom should be kept relatively low for easier manoeuvrability, better handling and lower power requirements for the tractor. Finally, any design decisions should be made on the basis of keeping the costs of altering a given baseline design to a minimum. We shall consider approaching all these objectives and/or constraints in three different formulations in Sections 4-6.

On the spectrum of optimization scopes, the exercise we report on here is closer to the local end of the scale. While the chosen subassemblies are optimized in a way that allows for the exploration of design spaces with multiple local optima (a global feature), we essentially aim to improve upon the dynamics of an existing design. Figure 3 depicts this original, baseline design, comprising a main frame (which rides directly on the wheels) and the spraying boom, sitting on a pair of air bellows, attached to the main frame through an intermediary mechanism.

Figure 4 peels parts of this assembly away in two steps to reveal the internal workings of the machine, as well as to highlight key references and notations, which will be of

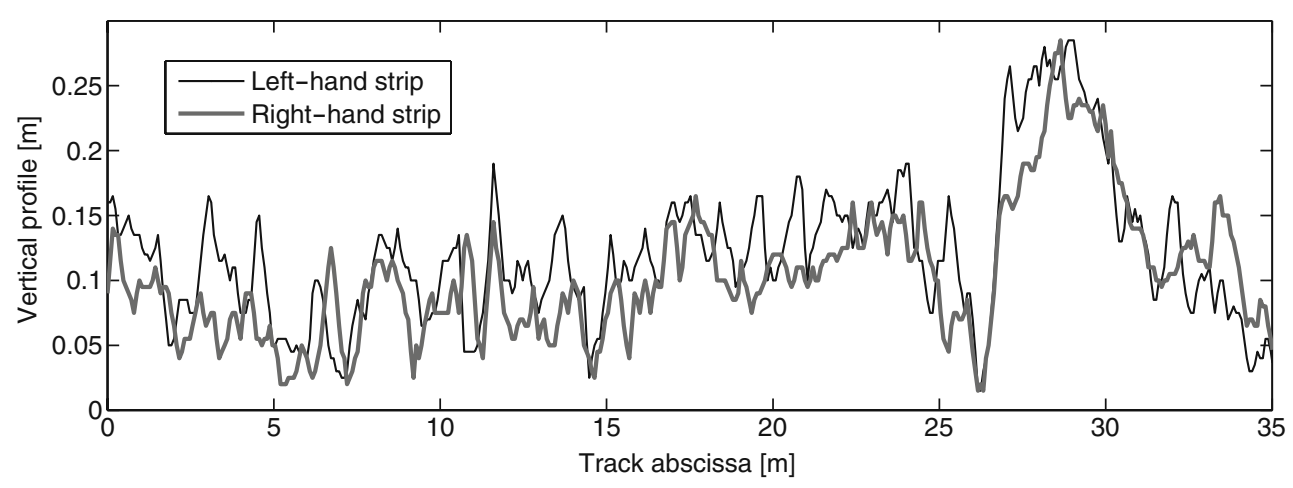

Fig. 2 The ISO 5008:2002 rough track 
Fig. 3 The sprayer boom and its mounting mechanism. Figure 4 offers a more detailed view of the main components, as well as some of the notations referred to in the text

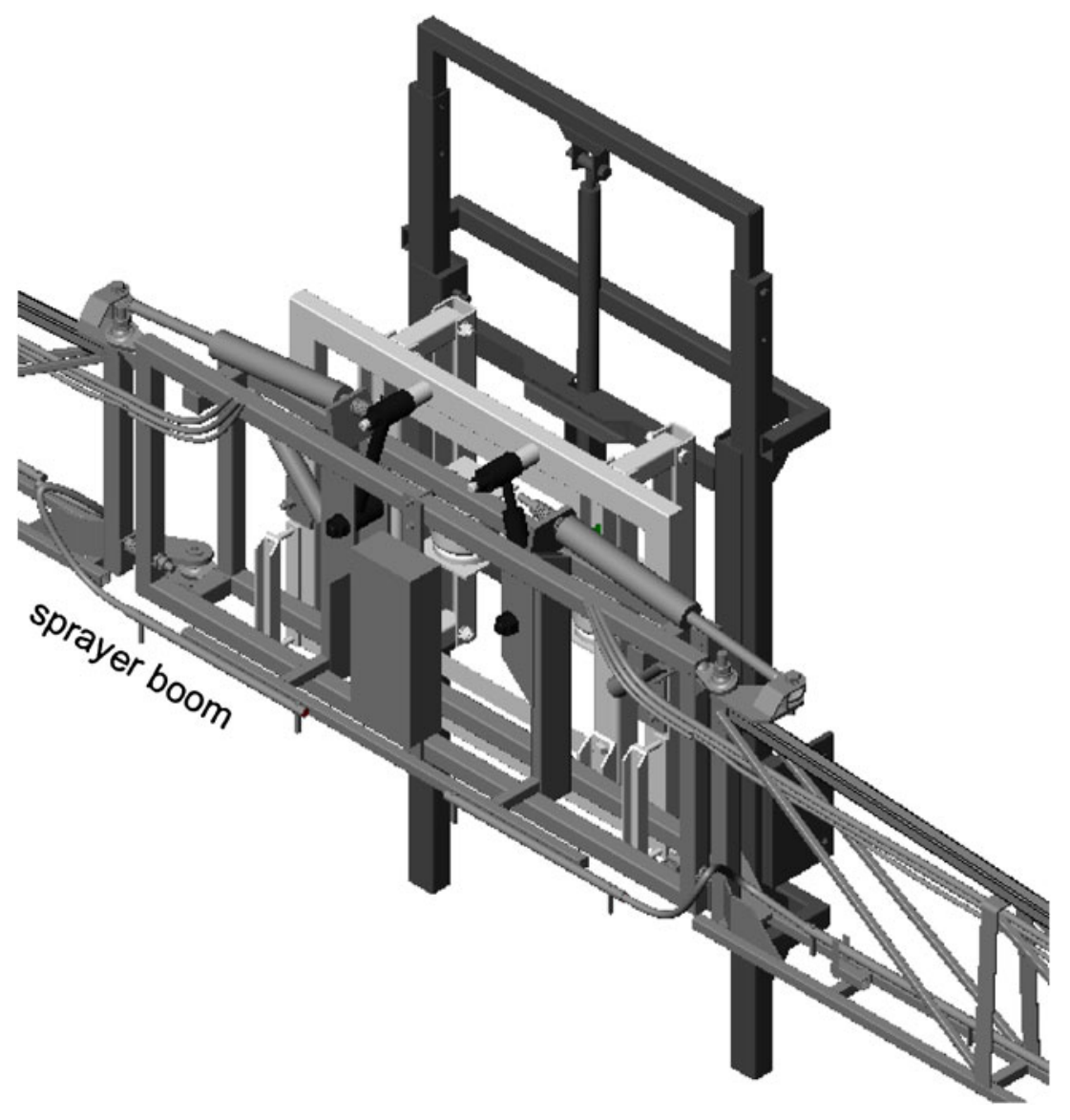

significance in the course of the forthcoming analysis of its dynamics.

To conclude this initial presentation of the object of our design study, the sketch in Fig. 5 aims to clarify the kinematics of the complete assembly_we shall consider all this in much more detail in the next section.

Finally, it is worth noting here that this type of problem is often solved by employing active control systems and/or strategically positioned dampers (see, for instance, the work of Anthonis et al. (2005), who examined the impact of the positioning of such dampers on the standard deviation of the absolute boom rotation around the horizontal axis, while the tractor covers a given track). Here, however, cost and complexity constraints (the third item on the 'wish-list') preclude the use of such means, forcing us to look at altering the shape of the structure itself as a means of improving its performance. In fact, we will show that what is perhaps the cheapest substantive modification, the optimization of the design of the trapezoidal linkage system that connects the boom to the intermediary structure, has an unexpectedly powerful impact on the damping of the rolling motion. First though, we need to consider some of the intricacies of the transmission of this motion between the main frame and the boom.

\section{Suspension dynamics}

Modeling the somewhat complex dynamics of the spraying machine essentially boils down to the following problem. As the wheels ride along the standard rough track, the asymmetric nature of the track causes the main frame of the machine to tilt by an angle $\varphi(t)$ (denoted simply as $\varphi$ in what follows, but continuing to mean a time-dependent variable)-see Fig. 6 for a definition of $\varphi$, the geometry of the wheels and the related coordinate systems. We seek the resulting spraying boom roll angle $\Psi(t)$ (also a function of time, though denoted simply as $\Psi$ in the following analysis). The other key variable needed for a full description of the motion of the boom is the variation of the length of the air bellows $S_{1} P_{1}=S_{2} P_{2}=L(t)$ (simply $L$ for the rest of this paper) as the machine is towed along the track.

We begin by formulating the equations of motion of the intermediary mechanism and the trapezoidal linkage system, whereby the boom is connected to it. The dimensions, forces and coordinate systems referred to in the following calculations are as illustrated in Figs. 4 and 7.

We write the equations describing the motion of the intermediary mechanism in the system defined by the axes $Y$ and 


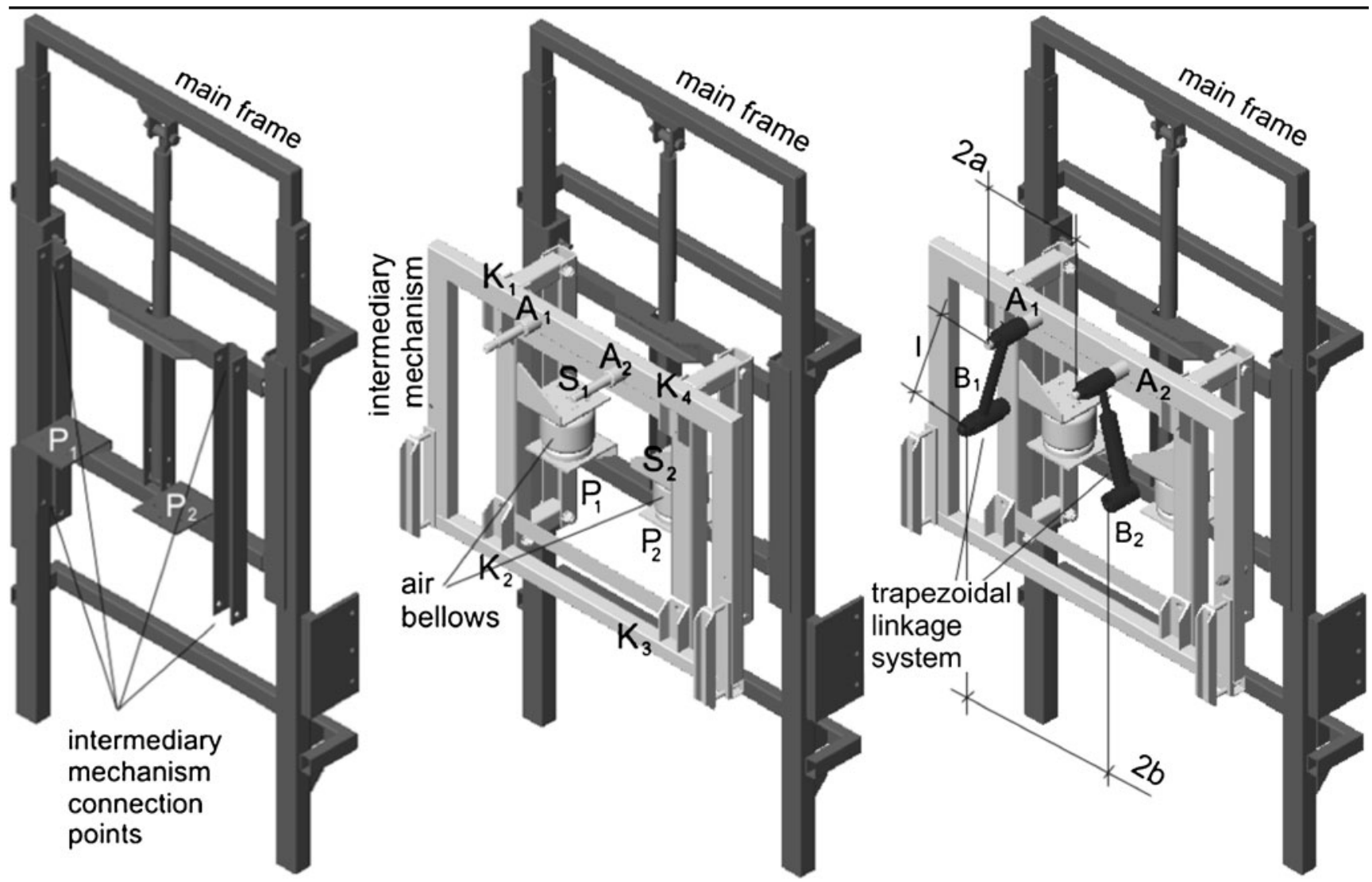

Fig. 4 Slice-by-slice view of the spraying machine suspension. From left to right: main frame, main frame plus the intermediary mechanism and main frame, intermediary mechanism, plus the trapezoidal linkage system, which holds the boom in place—see Fig. 3 for the complete assembly

$Z$ (with the origin in $P_{120}$-see Fig. 6 for its definition) $)^{2}$ as follows:

$$
\begin{gathered}
m_{1} \ddot{Y}_{C_{1}}=T_{1} \cos \left(\alpha_{1}+\varphi\right)+T_{2} \cos \left(\alpha_{2}+\varphi\right) \\
-2 E \sin \varphi-F_{1} l_{1} \sum_{i=1}^{4} \frac{Z_{3 K_{i}}}{l_{i}^{2}}
\end{gathered}
$$

and

$$
\begin{aligned}
m_{1} \ddot{Z}_{C_{1}}= & T_{1} \sin \left(\alpha_{1}+\varphi\right)+T_{2} \sin \left(\alpha_{2}+\varphi\right) \\
& +2 E \sin \varphi-m_{1} g+F_{1} l_{1} \sum_{i=1}^{4} \frac{Y_{3 K_{i}}}{l_{i}^{2}}
\end{aligned}
$$

where $Z_{3 K_{i}}$ and $Y_{3 K_{i}}$ are the vertical and horizontal coordinates respectively of point $K_{i}, i=1 \ldots 4$ with respect to the coordinate system $Y_{3} Z_{3}, Z_{C_{1}}$ is the vertical coordinate of the center of gravity of the intermediary mechanism, $m_{1}$ is the mass of the intermediary mechanism, $F_{1,2,3,4}$ are the forces acting on the intermediary mechanism in the

${ }^{2}$ The motion of the mechanism in the $\mathrm{X}$ direction is considered negligible. points $K_{1,2,3,4}, T_{1}$ and $T_{2}$ are the forces in the trapezoidal linkage system, $\mathrm{E}$ is the elastic force in the bellows and $l_{i}=\sqrt{Y_{3 K_{i}}^{2}+Z_{3 K_{i}}^{2}}$. Re-arranging, we obtain a pair of equations in terms of $\ddot{L}$, of the form:

$$
A_{3} \ddot{L}+C_{3}=T_{1} \cos \left(\alpha_{1}+\varphi\right)+T_{2} \cos \left(\alpha_{2}+\varphi\right)+F_{1} t_{1}
$$

and

$$
A_{4} \ddot{L}+C_{4}=T_{1} \sin \left(\alpha_{1}+\varphi\right)+T_{2} \sin \left(\alpha_{2}+\varphi\right)+F_{1} t_{2},
$$

where $A_{3}, A_{4}, C_{3}$ and $C_{4}$ are functions of $L, \dot{L}, \varphi, \dot{\varphi}, \ddot{\varphi}$, $\alpha_{1}, \alpha_{2}, a, b$ and $l$.

Moving on to the boom (whose geometry and the associated parameters referred to in the following calculations are shown in Fig. 8) we construct the differential equations describing its motion in a similar form:

$m_{2} \ddot{Y}_{C_{2}}=-T_{1} \cos \left(\alpha_{1}+\varphi\right)-T_{2} \cos \left(\alpha_{2}+\varphi\right)$,

$m_{2} \ddot{Z}_{C_{2}}=-T_{1} \sin \left(\alpha_{1}+\varphi\right)-T_{2} \sin \left(\alpha_{2}+\varphi\right)-m_{2} g$ 


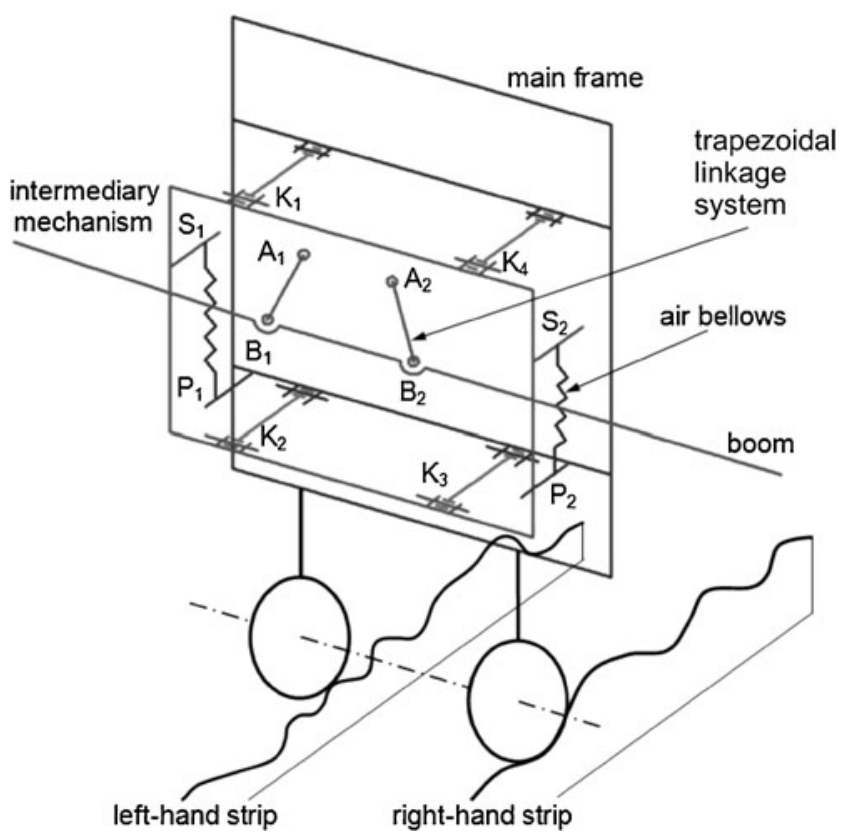

Fig. 5 Simplified kinematic sketch of the machine

and

$$
\begin{aligned}
J_{2} \ddot{\Psi}= & T_{1}\left[d_{2} \cos \left(\alpha_{1}+\varphi-\Psi\right)+b \sin \left(\alpha_{1}+\varphi-\Psi\right)\right] \\
& +T_{2}\left[d_{2} \cos \left(\alpha_{2}+\varphi-\Psi\right)+b \sin \left(\alpha_{2}+\varphi-\Psi\right)\right],
\end{aligned}
$$

where $m_{2}$ is the mass of the boom, $d_{2}$ is the distance between the segment $B_{1} B_{2}$ and the center of gravity of the boom $\left(C_{2}\right)$ on level ground at standstill and $J_{2}$ is the moment of inertia of the boom with respect to an axis parallel with the direction of travel through $C_{2}$.
Re-arranging as before, we obtain a set of equations in terms of $\ddot{L}$ and $\ddot{\Psi}$ of the form:

$A_{1} \ddot{L}+B_{1} \ddot{\Psi}+C_{1}=T_{1} \cos \left(\alpha_{1}+\varphi\right)+T_{2} \cos \left(\alpha_{2}+\varphi\right)$,

$A_{2} \ddot{L}+B_{2} \ddot{\Psi}+C_{2}=T_{1} \sin \left(\alpha_{1}+\varphi\right)+T_{2} \sin \left(\alpha_{2}+\varphi\right)$

and

$J_{2} \ddot{\Psi}=T_{1} e_{1}+T_{2} e_{2}$,

where $A_{1}, A_{2}, B_{1}, B_{2}, C_{1}$ and $C_{2}$ are, once again, functions of $L, \dot{L}, \Psi, \dot{\Psi}, \varphi, \dot{\varphi}, \ddot{\varphi}, \alpha_{1}, \dot{\alpha_{1}}, \ddot{\alpha}_{1}, \alpha_{2}, \dot{\alpha}_{2}, \ddot{\alpha}_{2}, a, b$ and l. Eliminating $T_{1}, T_{2}$ and $F_{1}$ we obtain a system of the form:

$\left\{\begin{array}{l}M_{1} \ddot{L}+P_{1} \ddot{\Psi}=Q_{1} \\ M_{2} \ddot{L}+P_{2} \ddot{\Psi}=Q_{2}\end{array}\right.$,

which we can solve for the second derivatives of the length $L$ of the air bellows and the roll angle $\Psi$ to obtain:

$\left\{\begin{array}{l}\ddot{L}=\left(Q_{1} P_{2}-Q_{2} P_{1}\right) /\left(M_{1} P_{2}-M_{2} P_{1}\right) \\ \ddot{\Psi}=\left(Q_{1} M_{2}-Q_{2} M_{1}\right) /\left(M_{1} P_{2}-M_{2} P_{1}\right)\end{array}\right.$.

Using the notation $u_{1}=L, u_{2}=\dot{L}, u_{3}=\Psi$ and $u_{4}=$ $\dot{\Psi}$ we can now form the following system of differential equations:

$\left\{\begin{array}{l}\dot{u_{1}}=u_{2} \\ \dot{u_{2}}=\left(Q_{1} P_{2}-Q_{2} P_{1}\right) /\left(M_{1} P_{2}-M_{2} P_{1}\right) \\ \dot{u_{3}}=u_{4} \\ \dot{u_{4}}=\left(Q_{1} M_{2}-Q_{2} M_{1}\right) /\left(M_{1} P_{2}-M_{2} P_{1}\right)\end{array}\right.$,
Fig. 6 Vertical cross section view (from behind) of the wheels of the machine riding along a flat surface (left) and along the two strips comprising the standard rough track (right)

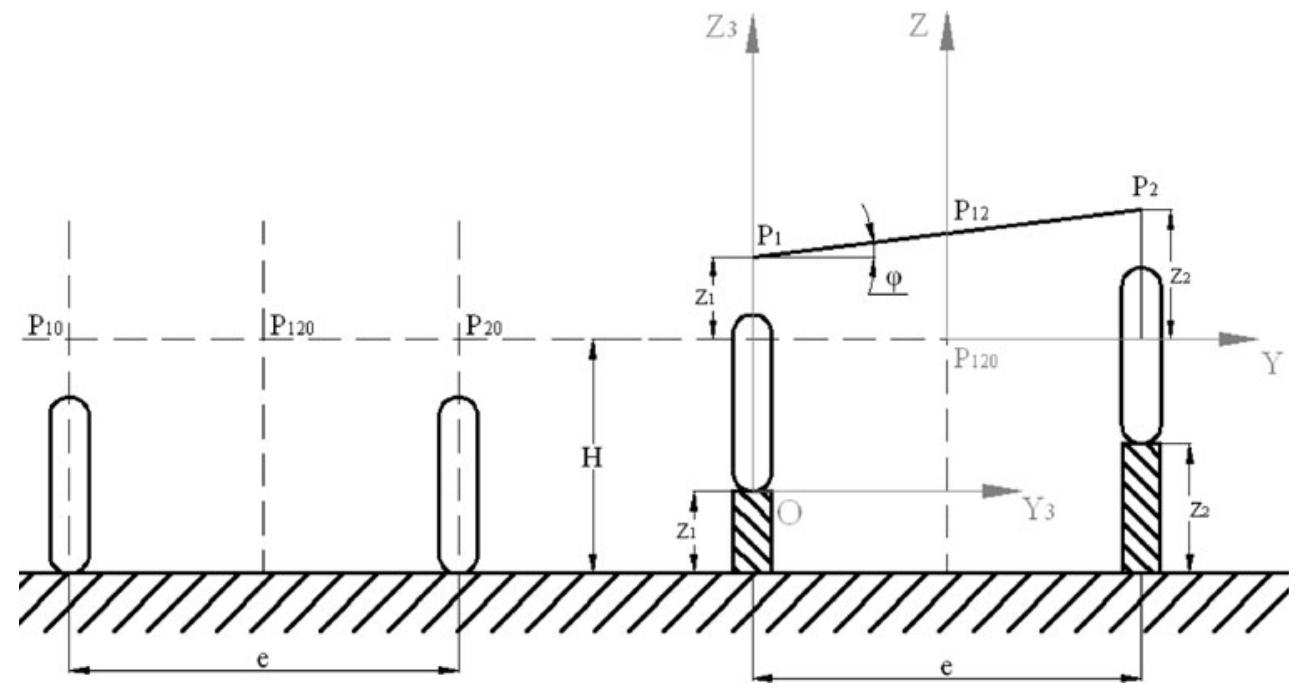




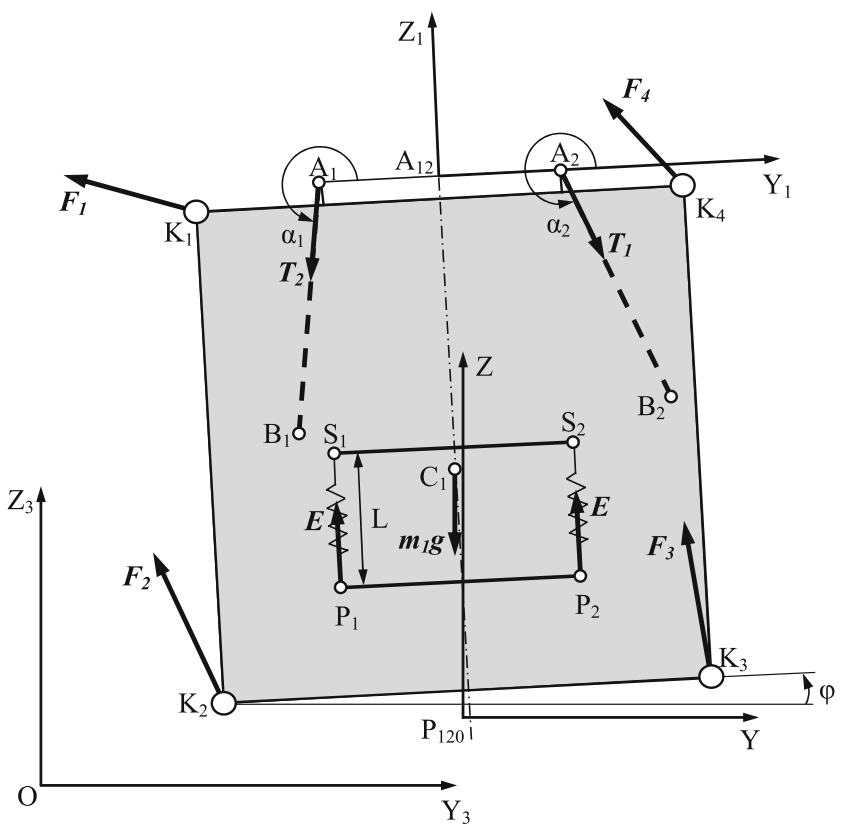

Fig. 7 Forces, angles and coordinate systems associated with the intermediary mechanism and the trapezoidal linkage system

which we solve using the Runge-Kutta numerical scheme, subject to the following boundary conditions at $t=0$ : $u_{1}=L_{0}$ (where $L_{0}$ is the length of the air bellows loaded by the weight of the boom) and $u_{2,3,4}=0$. We have thus obtained a numerical model linking $\Psi$ to $\phi$, which, as we indicated at the outset, is the key relationship defining the dynamic behavior of the system.

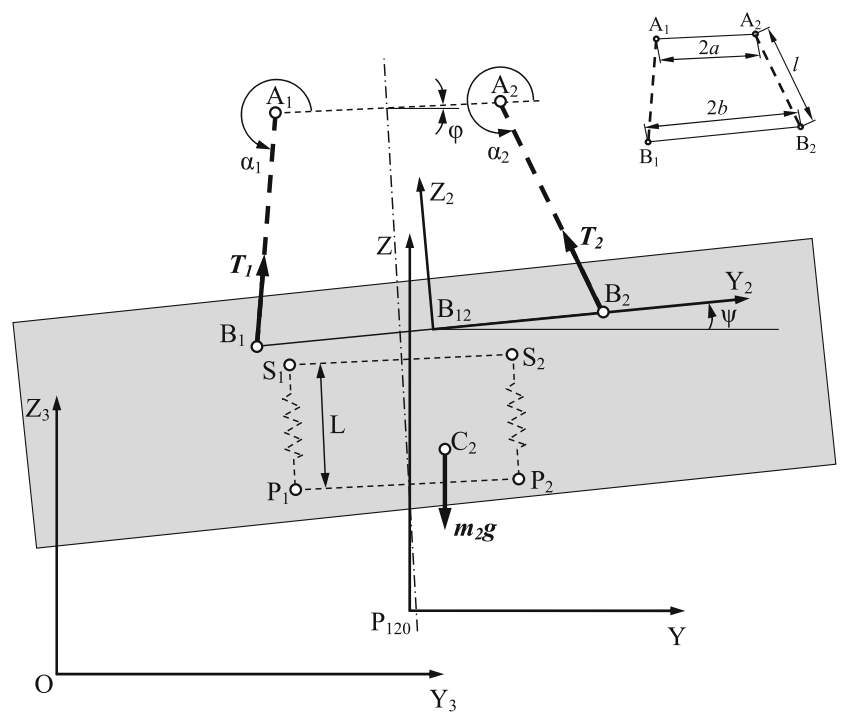

Fig. 8 Forces, angles and coordinate systems associated with the trapezoidal linkage system and the spraying boom

\section{Design subject to strict cost constraints}

Here is the design scenario we shall be considering in what follows. The manufacturer of the spraying equipment wishes to improve the spray deposition effectiveness of the machine, but, as a result of cost considerations, is only able to entertain the possibility of altering the shape of the linkage system.

The parameters that determine the geometry are $a, b$ and $l$, as highlighted in Fig. 4. Conceptually, therefore, this is a three-variable, continuous value problem. In practice, however, the problem is complicated slightly by the fact that one of the linkages includes a hydraulic cylinder (this can be used for in-situ length adjustments when operating on inclined terrain) and a discrete series of such cylinders is available. We therefore need a discrete valued design variable here (essentially a catalogue order number). The linkage length $l$ can take its values within a range determined by interference constraints, the travel of the hydraulic cylinder identified by the discrete variable and a constraint related to the ground clearance of the boom. Further, due to assembly constraints, the value of $l$ determines the range within which $b$ can take its values. The range of $a$ remains fixed. As a result, a total of four variables are needed (one discrete and three continuous) and some 'sleight of hand' is required in their definition - the evolutionary algorithm will actually be searching the space of a set of intermediate variables that define $a$ and $b$ with respect to their variable ranges-but ultimately the search yields an $a, b$ and $l$ triplet.

We sought to minimize the maximum roll angle recorded throughout the approximately $31.5 \mathrm{~s}$ journey of the machine along the standard rough track, that is we solved the optimization problem

$\min _{a, b, l}\left(\max _{t \in[0,31.5]} \Psi\right)$,

where $\Psi$, as a function of time, was estimated through a Runge-Kutta solution of the system (1) each time the evolutionary algorithm required the computation of the fitness of a putative individual. This yielded the values shown in Table 1 , including $\Delta h$, which is the maximum vertical travel

Table 1 Features of the baseline and optimized machine, including the maximum roll angle, reduced eightfold

\begin{tabular}{llllll}
\hline Variant & $a[\mathrm{~m}]$ & $b[\mathrm{~m}]$ & $l[\mathrm{~m}]$ & $\max \Psi$ & $\Delta h[\mathrm{~m}]$ \\
\hline Baseline & 0.1745 & 0.270 & 0.350 & $3.29^{\circ}$ & 0.603 \\
Optimized & 0.035 & 0.3185 & 0.397 & $0.4^{\circ}$ & 0.074 \\
\hline
\end{tabular}




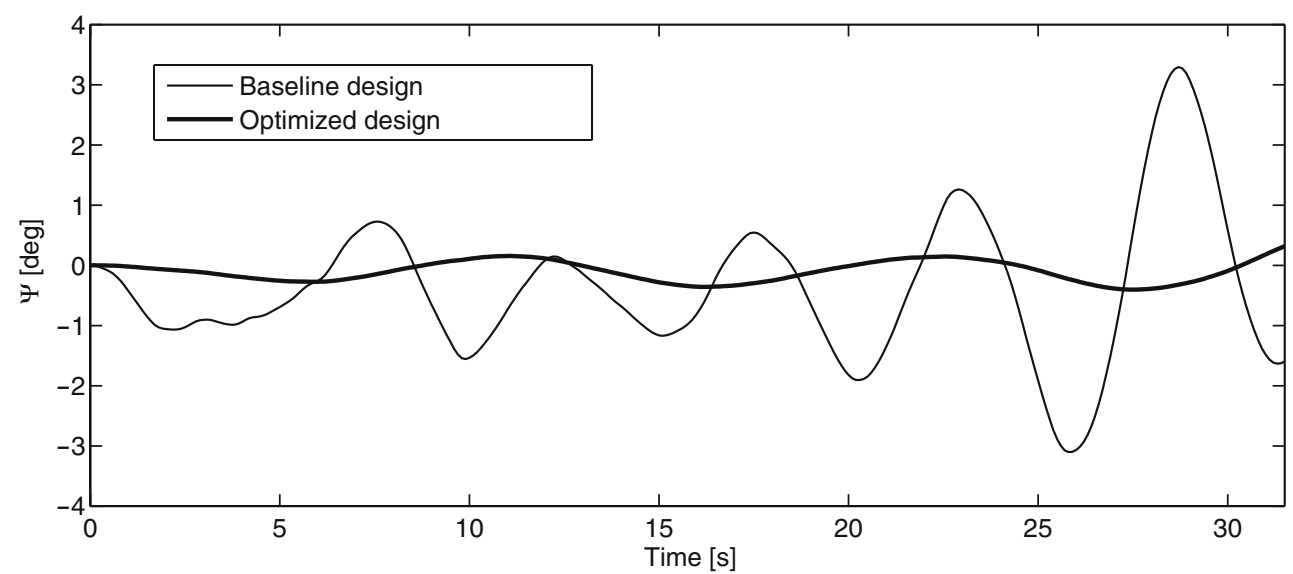

Fig. 9 Variation of the roll angle with time, as the machine travels along the track: the optimization of the trapezoidal linkage system leads to a clear improvement

of the tip of the boom (with respect to the value measured on level ground, at standstill), as the tractor follows the rough track. It is worth noting here that the baseline value $\Delta h=0.603 \mathrm{~m}$ exceeds the ground clearance of the machine $(0.5 \mathrm{~m}$, measured on level ground at standstill), that is, if the machine were driven along the standard track, the boom would strike the ground at that point. From the standpoint of the power of the evolutionary optimization, Fig. 9 is, perhaps, even more compelling. This shows the time variation of the roll angle for both the baseline and the optimized structure, highlighting the obvious improvement in the dynamic behavior of the equipment. This provides further evidence of what can be achieved by the mere alteration of the geometry of the trapezoidal linkage system.

\section{Design subject to relaxed cost constraints}

After looking at alterations of almost negligible cost, let us now consider some more expensive alternatives. Namely, we shall look at improving the geometry of the boom, still subject to certain cost-related constraints. More specifically, topology variations were constrained to structures constructed from a specified range of cell types. In other words, the evolutionary algorithm search is provided with components comparable to a set of Lego-bricks. Tooling constraints dictate that the Lego-bricks are geometrically similar amongst themselves.

We define a range of such cells, based on the topology of the original boom. As shown in Fig. 10, the boom
Fig. 10 The overall layout of the boom

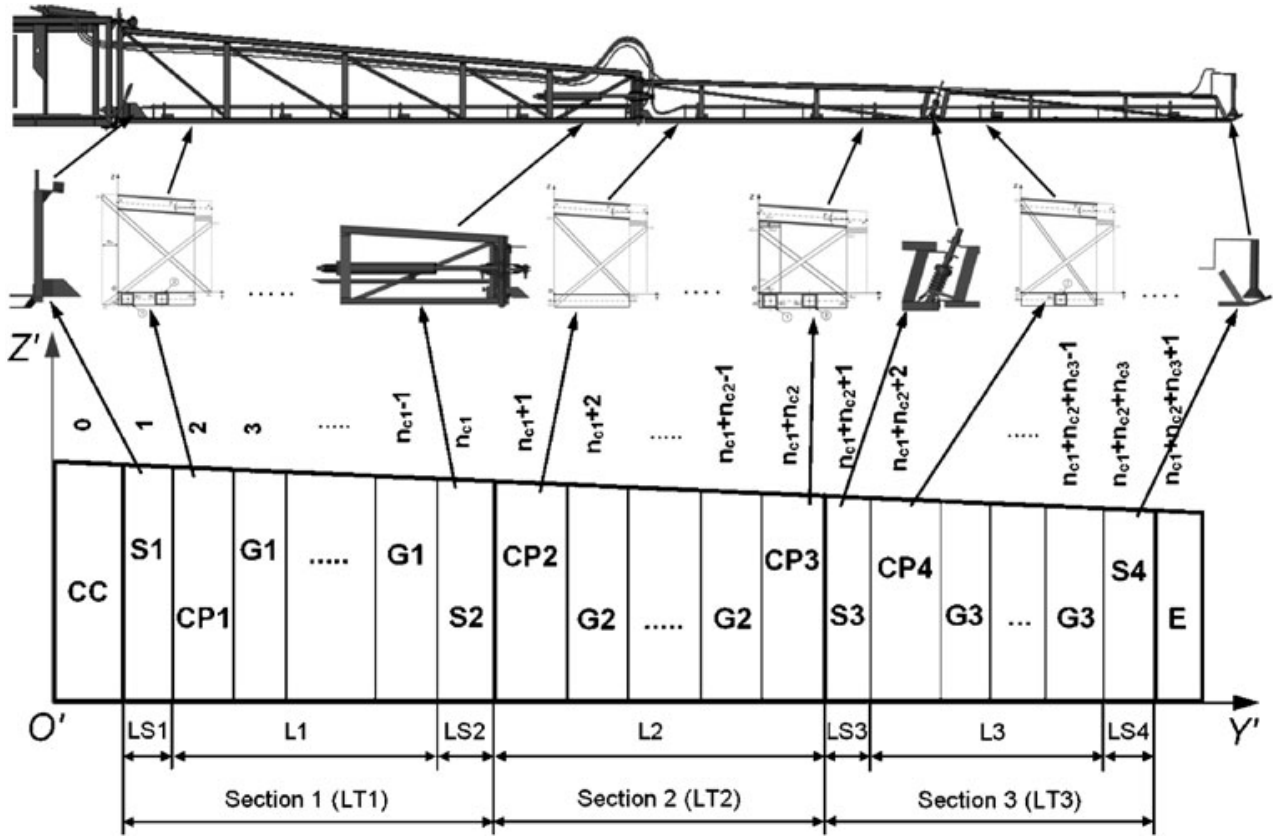


Fig. 11 G-type cell topology. The labels on the members correspond to those in Fig. 12
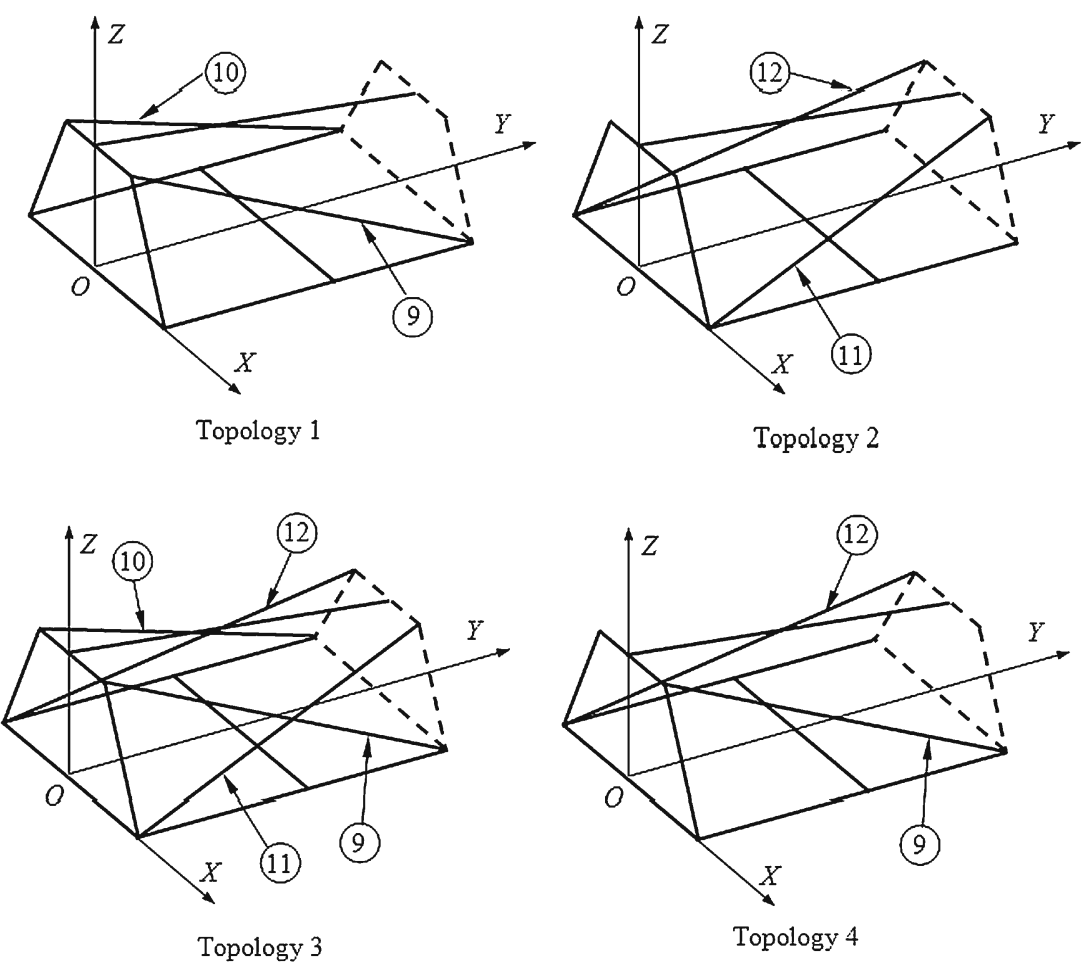

comprises three fundamental types of cells. First, G-type cells are labeled G1, G2 and G3 on the sketch, the number indicating which of the three sections of the boom each cell sequence belongs to. G-type cells come in four different topologies-these are shown in Fig. 11. Due to the manufacturing constraints mentioned above, each section can only feature one topology in its G-type cells, although it can have a variable number of them. Figure 12 shows a more detailed view of such a cell. They are, essentially, frameworks of trusses with a variety of cross-sections. At the base lie the four main structural components, elements 1 through 4, featuring large, hollow square cross sections.
Members 5, 6 and 7 have filled, flat, rectangular crosssections. The number 8 denotes the upper spanwise spine of the structure-it has once again a hollow square cross section, though larger than elements 1 through 4. Four diagonal members (9 through 12) complete the structure, featuring a small hollow square cross section. The variations in these last four members determine determine the topology of a given G-type cell, with topology 3 featuring all of them and the other topologies each including two of the four.

Second, the boom contains a number of S-type cells (S1 through S4, CC and E in Fig. 10), which cannot be altered
Fig. 12 The construction of a G-type cell. The labels on the members correspond to those in Fig. 11
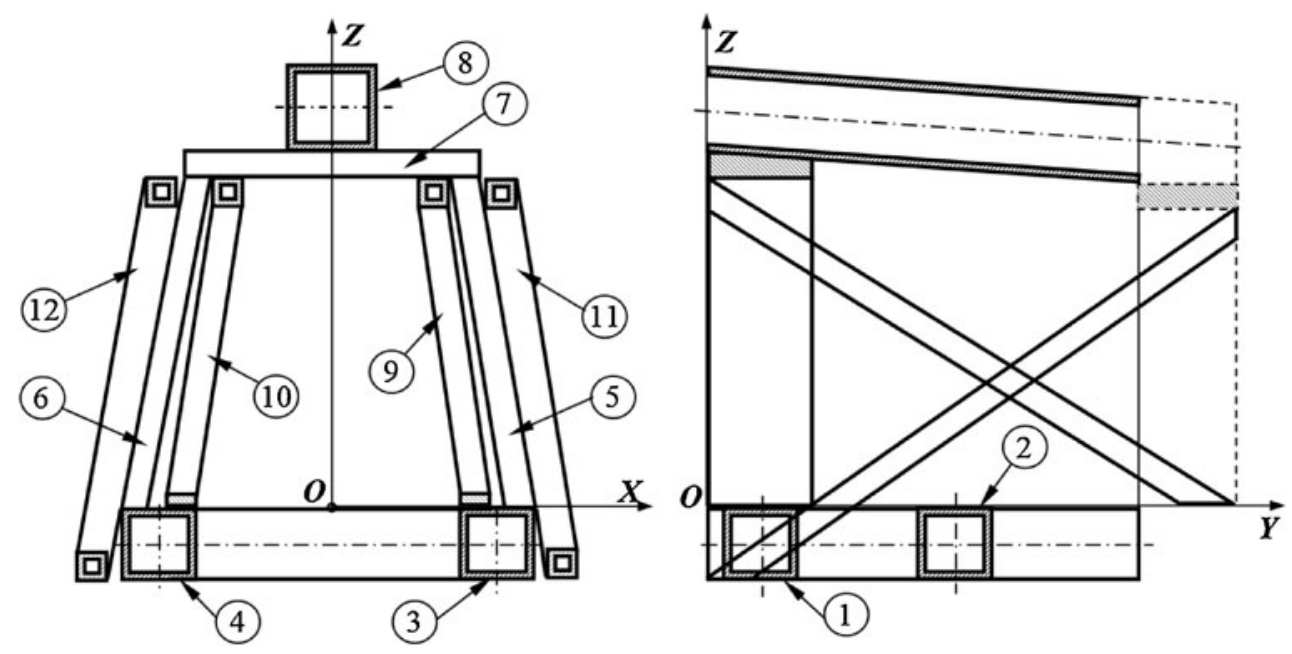
Table 2 Features of the baseline and the second optimized machine

\begin{tabular}{lllllll}
\hline Variant & $a[\mathrm{~m}]$ & $b[\mathrm{~m}]$ & $l[\mathrm{~m}]$ & $\max \Psi$ & $\Delta h[\mathrm{~m}]$ & Mass $[\mathrm{kg}]$ \\
\hline Baseline & 0.1745 & 0.270 & 0.350 & $3.29^{\circ}$ & 0.603 & 405.06 \\
Optimized & 0.035 & 0.320 & 0.395 & $0.28^{\circ}$ & 0.051 & 442.11 \\
\hline
\end{tabular}

A further reduction in the amplitude of the rolling motion is achieved, but at the expense of an overall weight gain

by the optimization process as they serve specific functional purposes (they connect adjacent sections, hold parts of the spraying ducting, etc.).

Third, there are CP-type cells, which are similar to Gtype cells and are derived through the removal of one or several of their members. As in the case of the G-type cells, each section of the boom can only feature one type of $\mathrm{CP}$ cell.

Here is how we defined the 'genetic make-up' of the variable topology boom:

- Variables 1 through 4: Trapezoidal linkage system geometry, as before (mixed types).

- Variable 5: Number of cells in Section 1 (taking integer values between 5 and 8 ).

- Variable 6: Number of cells in Section 2 (taking integer values between 3 and 6 ).

- Variable 7: Number of cells in Section 3 (taking integer values between 4 and 7).

- Variable 8: Topology of G-type cells in Section 1 (taking integer values between 1 and 4).

- Variable 9: Topology of G-type cells in Section 2 (taking integer values between 1 and 4).

- Variable 10: Topology of G-type cells in Section 3 (taking integer values between 1 and 4).
Table 3 Features of the baseline and the third optimized machine

\begin{tabular}{lllllll}
\hline Variant & $a[\mathrm{~m}]$ & $b[\mathrm{~m}]$ & $l[\mathrm{~m}]$ & $\max \Psi$ & $\Delta h[\mathrm{~m}]$ & Mass [kg] \\
\hline Baseline & 0.1745 & 0.270 & 0.350 & $3.29^{\circ}$ & 0.603 & 405.06 \\
Optimized & 0.035 & 0.2825 & 0.445 & $0.43^{\circ}$ & 0.079 & 396.92 \\
\hline
\end{tabular}

The evolutionary algorithm search has shaven approximately $8 \mathrm{~kg}$ off the weight of the boom

Table 2 shows the results of the optimization study over the design space defined by the variables listed above. The maximum roll angle has now been reduced even further, but the cost of this improvement is measured in the extra weight: the boom is now heavier than the baseline design by about $9 \%$. More importantly, we are now clearly down to very small changes along the range of rolling angles-this prompted us to investigate the extent to which the requirement to minimize max $\Psi$ could be relaxed. We discuss this in the next section.

\section{Design for high performance}

Our final study approaches the problem from a completely different perspective. The question is, could we reduce the weight of the boom, while still maintaining an acceptable maximum roll angle? The first element of this problem is the definition of an acceptable roll angle. In order to obtain this, we examined the geometry of the spraying process. As the sketch in Fig. 13 indicates, at a roll angle of zero and on level ground, every point under the boom falls within the spraying cone of a maximum of three nozzles. As the roll angle $\Psi$ increases, a critical point will appear eventually (when the overlap area shown in the sketch narrows to a
Fig. 13 Determining the maximum allowable roll angle: each point on the surface must be covered by the spraying cone of three adjacent nozzles
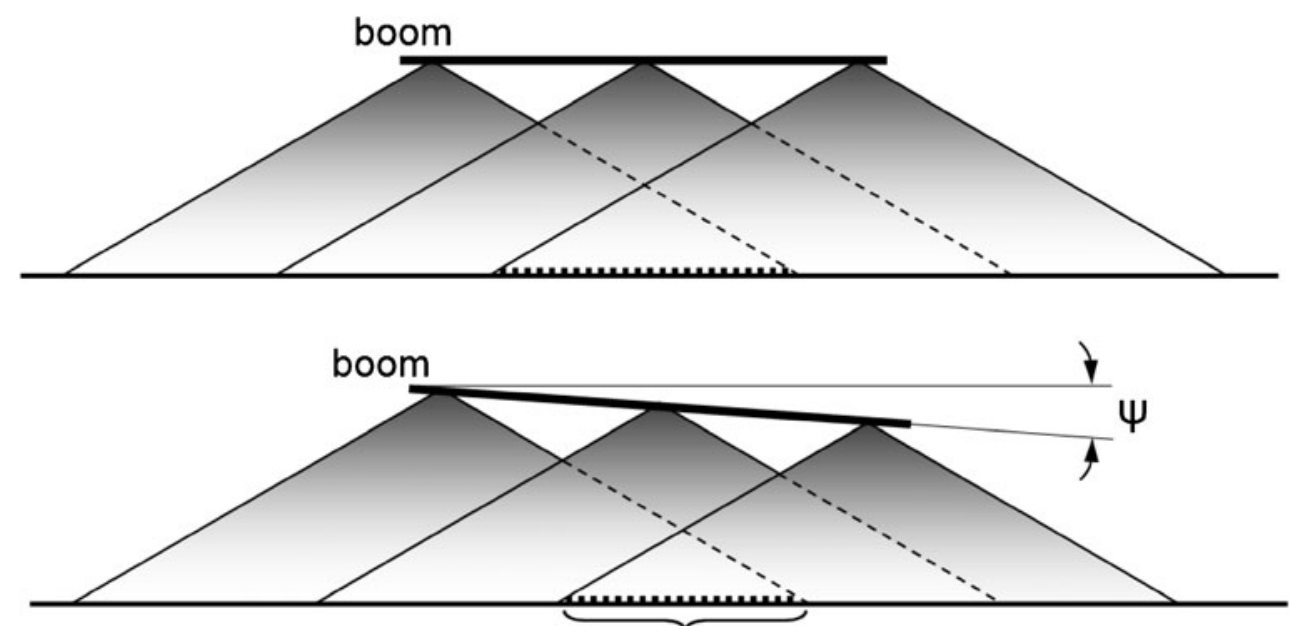

this overlap must not vanish 


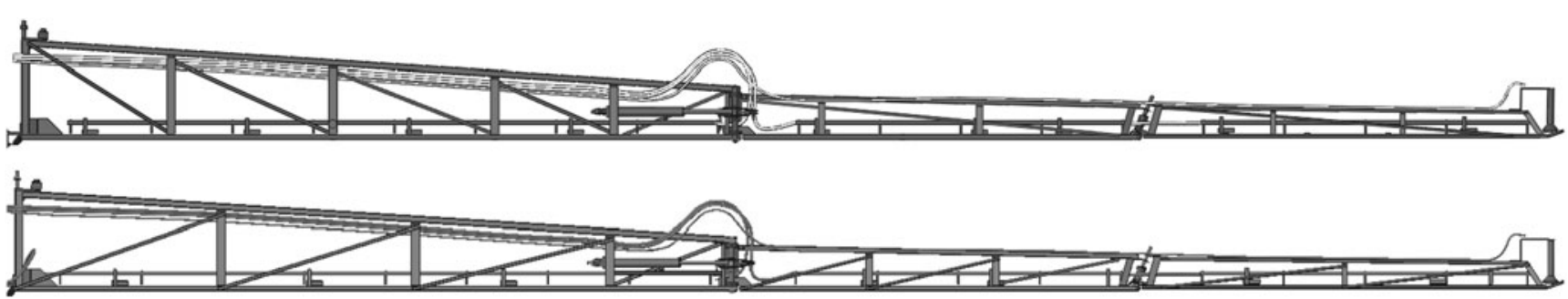

Fig. 14 The topology of the baseline boom (top) and the topology of the optimized boom (bottom)

point), beyond which there will be points on the ground that can only be reached by one nozzle. We have chosen this critical point as the rolling angle threshold (based on the constraint that effective spray deposition requires coverage by at least two nozzles) - in the case of the equipment in question, this value turns out to be $1^{\circ} 12^{\prime}$-we elected to use a more conservative $1^{\circ}$, giving us a margin of safety of about $20 \%$.

Therefore the mass was chosen as the objective function of the evolutionary algorithm optimization process here, with a constraint placed on the design space to the effect that $\max \Psi$ should remain below $1^{\circ}$. Table 3 lists the results of this final search, indicating that an 8-kg weight saving can be achieved, while satisfying the rolling angle constraint. Figure 14 compares the original topology with that of the minimum mass boom, while Fig. 15 shows a comparison of the roll angle variations of all three booms (note the small sacrifice that had to be made in terms of the maximum roll angle when minimizing the mass of the boom). We note here that a numerical stress analysis was performed on this lightened boom and this resulted in an acceptable factor of safety (the yield strength of the steel used in the manufacture of the boom is $240 \mathrm{MPa}$ and the maximum von Mises stress computed was $60 \mathrm{MPa}$ ).

\section{Conclusions and future work}

Nearly half a century after their inception, evolutionary algorithms as optimization tools have now reached a level of maturity, where they rank amongst the most powerful heuristics available to engineers wishing to explore large search spaces. The case study presented in this paper serves as further evidence of their power and versatility. We have shown that not only can they explore mixed design spaces (that is, design spaces featuring continuous, as well as discrete variables) effectively, but, via appropriate variable setups, they can be used as shape-, as well as topological optimization tools too, even when 'real-world' complications (such as intricate relationships between the ranges of various variables) would curtail other types of search methods. It is worth considering the obvious alternative here, the wider family of hill climber type algorithms. As the various cases of our design problem feature both continuous and discrete variables, gradient-based methods from this class would not be suited. Some success has been reported in the past on similar problems with other local heuristics, but the sheer size of the search space, as well as the inherent uncertainty about the multi-modality of the landscapes (would one need to run multiple parallel restarts?) once again
Fig. 15 Variation of the roll angle with time, as the machine travels along the track: the results of the second and the third optimization study

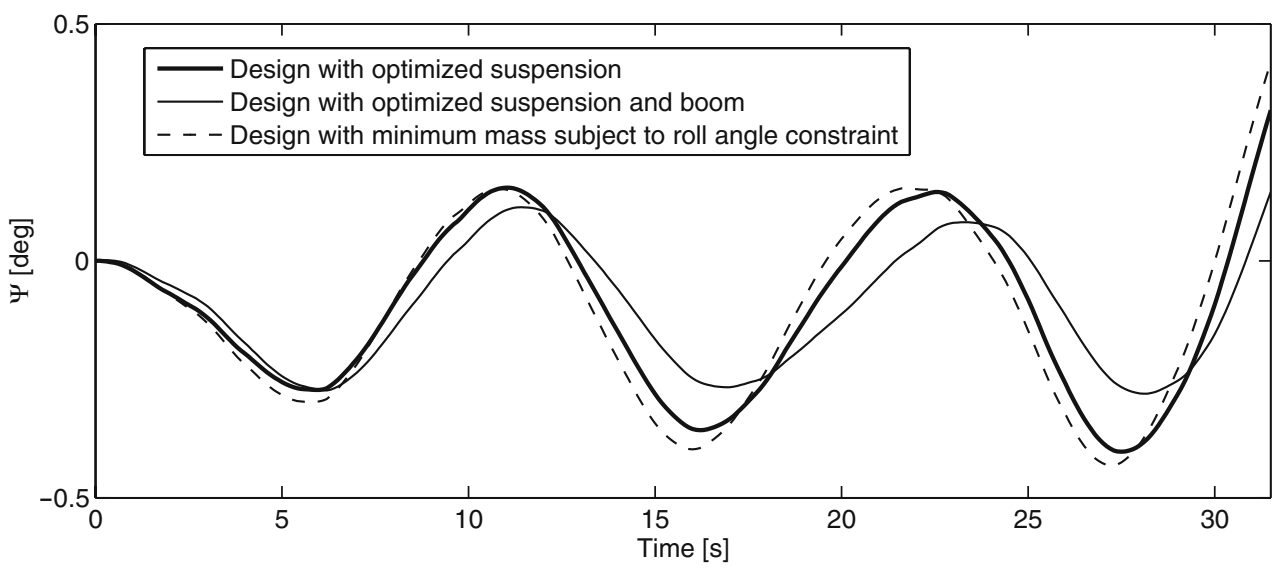


underscores the advantages of the evolutionary framework used here. Finally, the objectives discussed here are subject to a wide range of constraints-in fact, the mere identification of a feasible region (say, for the placement of a starting point for a local search heuristic) is a challenge in itself. The specially adapted evolutionary heuristic used here copes with this obstacle through its multi-stage design.

We tackled a structural optimization problem along three separate lines of attack and we have shown that in each case improvements were possible. In particular, the first study has practically eliminated a fundamental flaw in the design of an existing product-moreover, this was achieved through shape optimization, the results of which can be implemented at very low cost. At the higher cost end we have shown that structural weight reductions are possible without jeopardizing the dynamic performance of the equipment.

The methodology followed here is, of course, not specific to the type of machinery examined here. In fact, other types of truss structure could be parameterized and optimized in the way described here, by extending the concept of the generic cell templates introduced in Section 5 (that is, in a way analogous to our definition of a G-type cell) and variations on these (our CP-cells), as well as cells constrained by manufacturing considerations. This type of geometry description allows variations in topology, as well as in shape, without the need for specialized algorithms developed for particular objective functions.

Possible future extensions of the work described here include examining the possibilities afforded by relaxations of the cost constraints. This would allow, for instance, the use of a more extensive library of cells and cell topologies, the use of members of different cross-sections, etc.

On a more practical note, we have set out (and are in the process of completing) a programme of work aimed at the experimental analysis and validation of the results obtained here. We have designed a range of structural dynamics experiments on scale models of the ramps, the results of which, combined with the computational analysis reported on here, should provide an evidence base that will pave the way towards an industrial implementation of the work. We shall describe these experiments in a future paper.

Acknowledgments The work of L. Tudose and C. Stănescu was supported by Romanian Government grant PN II IDEI 2007-2010, CNCSIS Code ID-1077. The Royal Academy of Engineering and the Engineering and Physical Sciences Research Council (EPSRC) supported A. Sóbester's work.

\section{References}

Anthonis J, Audenaert J, Ramon H (2005) Design optimization for the vertical suspension of a crop sprayer boom. Biosyst Eng 90(2): 153-160

Clijmans L, Swevers J, De Baerdemaeker J, Ramon H (2000) Sprayer boom motion-part 1: derivation of the mathematical model using experimental system identification theory. J Agric Eng Res 76: 61-69

Dylejkoa PG, Kessissoglou NJ, Tso Y, Norwood CJ (2007) Optimisation of a resonance changer to minimise the vibration transmission in marine vessels. J Sound Vib 300:101-116

Fiacco AV, McCormick GP (1968) Nonlinear programming: sequential unconstrained minimization techniques. Wiley

Goldberg D (1989) Genetic algorithms in search, optimization and machine learning. Addison-Wesley Professional

Huang X, Xue YM (2010) A further review of eso type methods for topology optimization. Struct Multidisc Optim 41:671-683

ISO 5008:2002 (2002) Agricultural wheeled tractors and field machinery-measurement of whole-body vibration of the operator. International Standardization Organisation

Kamesh D, Pandiyan R, Ghosal A (2010) Modeling, design and analysis of low frequency platform for attenuating micro-vibration in spacecraft. J Sound Vib 329(17):3431-3450

Keane AJ (1995) Passive vibration control via unusual geometries: the application of genetic algorithm optimization to structural design. J Sound Vib 185(3):441-453

Keane AJ, Nair PB (2005) Computational approaches for aerospace design. Wiley

Langenakens J, Clijmans L, Ramon H, De Baerdemaeker J (1999) The effect of vertical sprayer boom movements on the uniformity of spray distribution. J Agric Eng Res 74:281-291

Martins JRRA, Poon NMK (2005) On structural optimization using constraint aggregation. In: 6th world congress on structural and multidisciplinary optimization. ISSMO, Rio de Janeiro, Brazil

Oka SY, Songa J, Park KS (2008) Optimal design of hysteretic dampers connecting adjacent structures using multi-objective genetic algorithm and stochastic linearization method. Engineering structures, vol 30, no $1240-1249$

París J, Navarrina F, Colominas I, Casteleiro M (2009) Topology optimization of continuum structures with local and global stress constraints. Struct Multidisc Optim 39:419-437

París J, Martínez Navarrina F, Colominas I, Casteleiro M (2010) Topology optimization of stuctures with stress constraints: aeronautical applications. In: IOP conf. series: materials science and engineering, vol 10, no 1-9

Parloo E, Guillaume P, Anthonis J, Heylen W, Swevers J (2005) Modelling of sprayer boom dynamics by means of maximum likelihood identification techniques-part 2: sensitivity-based mode shape normalization. Biosyst Eng 83(3):291-298

Sigmund O (2001) A 99 line topology optimization code written in matlab. Struct Multidisc Optim 21:120-127

Tudose L, Buiga O, Stefanache C, Sóbester A (2010) Automated optimal design of a two-stage helical gear reducer. Struct Multidisc Optim 42:429-435

Zhu JH, Zhang WH, Qiu KP (2007) Bi-directional evolutionary topology optimization using element replaceable method. Comput Mech 40:97-109 